\title{
Surrogate Assisted Design Optimization of an Air Turbine
}

\author{
Rameez Badhurshah and Abdus Samad \\ Department of Ocean Engineering, Indian Institute of Technology Madras, Chennai 600036, India \\ Correspondence should be addressed to Abdus Samad; samad@iitm.ac.in
}

Received 30 May 2014; Revised 17 September 2014; Accepted 27 September 2014; Published 14 October 2014

Academic Editor: Farid Bakir

Copyright (C) 2014 R. Badhurshah and A. Samad. This is an open access article distributed under the Creative Commons Attribution License, which permits unrestricted use, distribution, and reproduction in any medium, provided the original work is properly cited.

\begin{abstract}
Surrogates are cheaper to evaluate and assist in designing systems with lesser time. On the other hand, the surrogates are problem dependent and they need evaluation for each problem to find a suitable surrogate. The Kriging variants such as ordinary, universal, and blind along with commonly used response surface approximation (RSA) model were used in the present problem, to optimize the performance of an air impulse turbine used for ocean wave energy harvesting by CFD analysis. A three-level full factorial design was employed to find sample points in the design space for two design variables. A Reynolds-averaged Navier Stokes solver was used to evaluate the objective function responses, and these responses along with the design variables were used to construct the Kriging variants and RSA functions. A hybrid genetic algorithm was used to find the optimal point in the design space. It was found that the best optimal design was produced by the universal Kriging while the blind Kriging produced the worst. The present approach is suggested for renewable energy application.
\end{abstract}

\section{Introduction}

A computational fluid dynamics (CFD) based design requires long time to evaluate objective functions of a problem. This type of analysis is called high-fidelity analysis and any high-fidelity model requires many numerical simulations to evaluate the functions. To optimize a CFD system, physicsbased low-fidelity models or surrogate models or surrogates are employed and these help in reducing computational burden. A global optimization algorithm, such as hybrid genetic algorithm (HGA), which mimics the evolutionary principle assisted by sequential quadratic programming helps in finding the optimal design [1].

The low-fidelity models depend on the nature of the problem while the model-accuracy depends on several factors such as nature and distribution of sampling points, type of model, optimizer capability, and number of variables. It was found that the Kriging (KRG) models were as competitive as other surrogates [2]. There are the surrogates such as response surface approximation (RSA) and artificial neural network (ANN), and these surrogates further have their variants. Researchers are trying to increase the effectiveness and accuracy in constructing the surrogates, to increase the number of variables, to reduce the number of design points, and so forth [3]. Hence it is imperative to bring the researches to application level and check whether particular surrogate or surrogate variants can perform better than the others.

There are ample articles describing surrogate models and their applicability to different problems [4-6]. The models are problem dependent and selecting a proper model requires designer expertise [4]. Peter and Onera [4] compared the surrogate models in an industrial context to design a stator blade in order to optimize the local pressure at the exit and showed that universal Kriging (UKR) provided the better results in terms of approximating the exact function. In some other application, it did not perform well as compared to the other surrogates [2]. The KRG variants such as ordinary Kriging (OKR), UKR, and blind Kriging (BKR) which performed well in several applications for optimization were compared for analytical function based optimizations [2]. BKR performed better in several applications [2]. But there was no reported application in CFD or turbomachinery application found in the literatures regarding the applicability of BKR.

One of the renewable energy systems is oscillating water column (OWC) based system which harvests energy from the ocean waves. OWC is a partially submerged hollow cylindrical column consisting of a bidirectional flow turbine or simply a bidirectional air turbine installed inside the column [7]. The periodic wave pattern produces a reciprocating air flow over the turbine and thus it rotates the turbine in a 


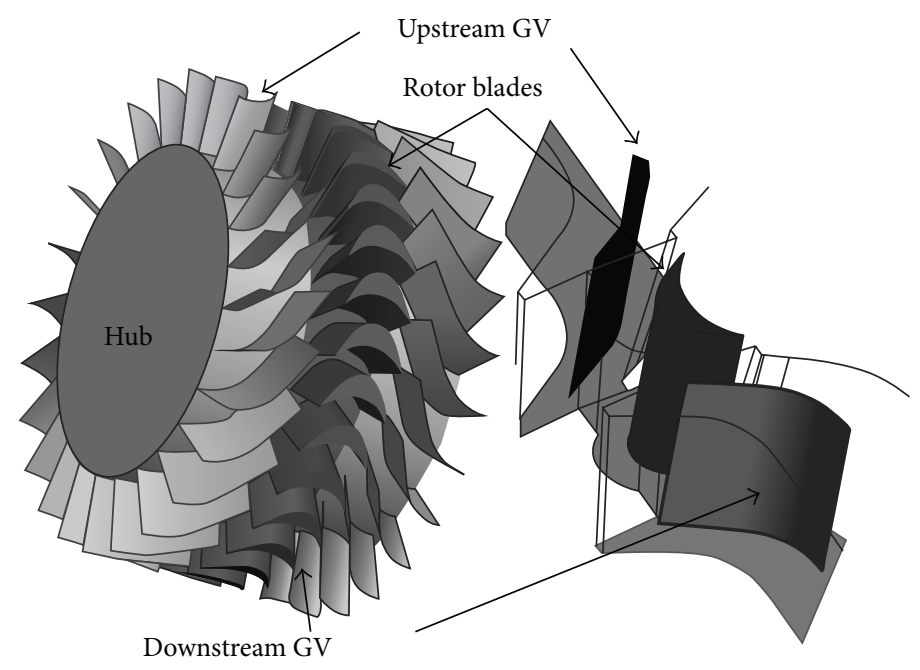

(a) $3 \mathrm{D}$ view

(b) Periodic view

FIGURE 1: Impulse turbine.

single direction. The popular bidirectional turbines are either reaction or impulse type. The impulse turbine is better in terms of having higher operating range. The impulse turbine has a symmetrical rotor sandwiched between two sets of guide vanes (GVs). The GVs essentially deflect the flow through the inlet and helps increase the kinetic energy of flow. As a result, the fluid particle hits the rotor blade (RB) and gives an "impulse" to the turbine [7]. The earliest reported systematic optimization study was on reaction turbine airfoil blade shape modification for achieving multiple objectives [8]. The optimization of rotor blade sections of an impulse turbine was carried by Gomes et al. [9].

The present work compares the Kriging variants along with RSA surrogate to enhance the efficiency of the turbine used for wave energy extraction. Numbers of blade and guide vanes were modified to enhance the efficiency of the turbine. Numerical analysis to validate the performance of the surrogates was implemented in this problem. Detailed numerical approach and surrogate strategy along with results have been discussed in this paper.

\section{Problem Description and Numerical Procedure}

2.1. Model Description and CFD Methodology. Bidirectional flow impulse turbine was chosen as the reference geometry [10] for studying the applicability of surrogate techniques to turbomachinery application. Figure 1(a) shows the complete geometry. The turbine has 30 rotor blades $\left(N_{\mathrm{rb}}=30\right)$ and 26 guide vanes $\left(N_{\mathrm{gv}}=26\right)$ on either side of the rotor blade (RB). To reduce computational cost, simulation was run over single RB rather considering all the blades. Figure 1(b) shows the flow domain. The major specifications are listed in Table 1. To analyze the flow over the entire fluid domain (including all the blades), a higher computational cost is required. Hence, the walls of the domain where it faces the domain of other blades are given as periodic conditions. This has also been reported in the literature [11].
TABle 1: Design specifications of rotor and guide vane.

\begin{tabular}{ccc}
\hline & Parameter & Specifications \\
\hline Rotor blade profile & Circular-elliptical \\
Number of rotor blades $\left(N_{\mathrm{rb}}\right)$ & 30 \\
Rotor blade pitch & $26.7 \mathrm{~mm}$ \\
Hub diameter & $210 \mathrm{~mm}$ \\
Tip diameter & $298 \mathrm{~mm}$ \\
Tip clearance & $1 \mathrm{~mm}$ \\
Chord length $\left(l_{\mathrm{rb}}\right)$ & $54 \mathrm{~mm}$ \\
Blade inlet angle & $60^{\circ}$ \\
\hline Guide vane & Nuide vane profile & Plate type \\
& Stator blade pitch & 26 \\
Chord length $\left(l_{\mathrm{gv}}\right)$ & $30.8 \mathrm{~mm}$ \\
Tip clearance & $70 \mathrm{~mm}$ \\
Blade thickness & $1 \mathrm{~mm}$ \\
Guide vane inlet angle & $0.5 \mathrm{~mm}$ \\
\hline
\end{tabular}

The flow was computed over the passage of the upstream guide vane $(\mathrm{GV})$, then over the RB. Finally the flow leaves through the downstream GV. Steady state flow with frozen rotor approach was used. The turbulence model was $k-\varepsilon$ and the turbine rotated at constant low speed of $600 \mathrm{rpm}$. Inlet velocity $(v)$ was varied to compute flow over different flow coefficients and the exit pressure was set to $1 \mathrm{~atm}$. To achieve the uniform flow, the flow domain was extended to 8.5 times the chord length [11]. The Reynolds averaged Navier-Stoke (RANS) equations were solved for evaluating torque $(T)$ and total pressure drop $(\Delta P)$. Rather than expressing torque, pressure drop, and velocity directly, these were addressed in dimensionless forms as torque coefficient $\left(C_{t}\right)$, input power coefficient $\left(C_{\text {in }}\right)$, and flow coefficient $(\varphi)$, respectively. Flow coefficient $(\varphi)$ is the ratio of the inlet axial velocity to the 
circumferential velocity. The mathematical formulations of the parameters $[10,12]$ are

$$
\begin{gathered}
\text { Torque coefficient: } C_{t}=\frac{2 T}{\left\{\rho\left(v^{2}+U^{2}\right) b l_{\mathrm{rb}} N_{\mathrm{rb}} r\right\}}, \\
\text { Input power coefficient: } C_{\mathrm{in}}=\frac{2 \Delta P Q}{\left\{\rho\left(v^{2}+U^{2}\right) b l_{\mathrm{rb}} N_{\mathrm{rb}} v\right\}}, \\
\text { Efficiency: } \eta=\frac{T \omega}{\Delta P Q}=\frac{C_{t}}{\phi \cdot C_{\mathrm{in}}}, \\
\text { Flow coefficient }: \phi=\frac{v}{U}
\end{gathered}
$$

The governing Navier-Stokes transport equations are as follows.

Mass

$$
\frac{\partial(\rho u)}{\partial x}+\frac{\partial(\rho v)}{\partial y}+\frac{\partial(\rho w)}{\partial z}=0
$$

and momentum

$$
\begin{aligned}
& -\frac{\partial(P)}{\partial x}+\frac{\partial\left(\tau_{x x}\right)}{\partial x}+\frac{\partial\left(\tau_{y x}\right)}{\partial y}+\frac{\partial\left(\tau_{z x}\right)}{\partial z}=\operatorname{div}\left(\rho_{u u}\right), \\
& -\frac{\partial(P)}{\partial x}+\frac{\partial\left(\tau_{x y}\right)}{\partial x}+\frac{\partial\left(\tau_{y y}\right)}{\partial y}+\frac{\partial\left(\tau_{z y}\right)}{\partial z}=\operatorname{div}\left(\rho_{v u}\right), \\
& -\frac{\partial(P)}{\partial x}+\frac{\partial\left(\tau_{x z}\right)}{\partial x}+\frac{\partial\left(\tau_{y z}\right)}{\partial y}+\frac{\partial\left(\tau_{z z}\right)}{\partial z}=\operatorname{div}\left(\rho_{w u}\right) .
\end{aligned}
$$

Discretization of model was done in ICEM-CFD and the mesh in the domain is shown in Figure 2. Unstructured tetrahedral elements with finer meshing near the blade surface and tip clearance region were employed. ANSYS-CFX was used as a RANS solver. Two mixing planes, one at each of the interfaces of the stator-rotor regions, were used. Table 2 lists the initial and boundary conditions. The domain was discretized into number of cells and each cell represents a finite volume with a central node, at which the flow properties were evaluated. The tip clearance zone was modeled with finer mesh and it was assured that sufficient nodes should be there in radial direction.

The simulations were run on $3.4 \mathrm{GHz}$ core i7-3370 processor with $8 \mathrm{~GB}$ RAM. The average number of iterations for converged results for a single simulation run was approximately 1000 and average time was approximately 10 hours.

2.2. Objective Function and Design Variables. Utilizing energy from natural resources has to be carried out efficiently so that the losses associated can be reduced. Hence, for the current problem, the turbine efficiency $(\eta)$ of the impulse turbine (3) was chosen to be the objective function. Two design variables (DVs), namely, number of the stator blades $\left(N_{\mathrm{gv}}\right)$ and the number of the rotor blades $\left(N_{\mathrm{rb}}\right)$, were selected. A feasible design space was formed by the lower and upper limits of the variables as shown in Table 3. The variation in blade number changes its pitch, thus depending on the

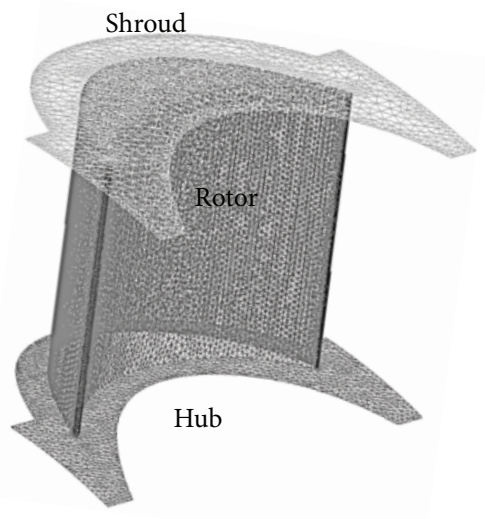

FIGURE 2: Mesh for rotor.

TABLE 2: Initial and boundary conditions.

\begin{tabular}{ll}
\hline Analysis & Steady state analysis \\
Fluid & Ideal gas \\
Turbulence model & $k-\varepsilon$ \\
Turbulence intensity & Medium $(5 \%)$ \\
Inlet boundary condition & Inlet axial speed \\
Rotational speed & $600 \mathrm{rpm}$ (constant) \\
Outlet boundary condition & Static pressure $(1 \mathrm{~atm})$ \\
Stage type & Frozen rotor \\
Domain & Periodic boundary \\
\hline
\end{tabular}

number of blades the fluid domain changes, and thus it affects turbine performance. More number of blades leaves lesser space for the fluid passage. As the inlet flow velocity is constant, the flow might get accelerated when it flows over more number of blades. This accelerated flow hits the rotor blade with more impulse, thus increasing the torque generated by the rotor. On the other hand, the same narrow passage can give flow blockage and can reduce performance.

2.3. Optimization Procedure. Optimization is central theme to any problem involving decision-making that involves choosing among alternatives. The measure of goodness of the alternatives is represented by the objective functions or performance indices [3]. Optimization methodology deals with the selection of the best alternative relative to the given designs.

The procedure for optimization is shown in Figure 3. Initially the design variables and the objectives are set up. As the surrogates cannot generate the initial population, a high-fidelity analysis like the CFD is carried out. The design variables lower and upper bounds are defined. At each of the cross combination of the DV's, CFD analysis is carried out. This being the initial data is fed to train the surrogate. After training the surrogate, the optimum point is searched using an optimizer. Once the convergence criteria are achieved, the surrogate predicted results are cross validated with CFD; else the design space is further modified. 


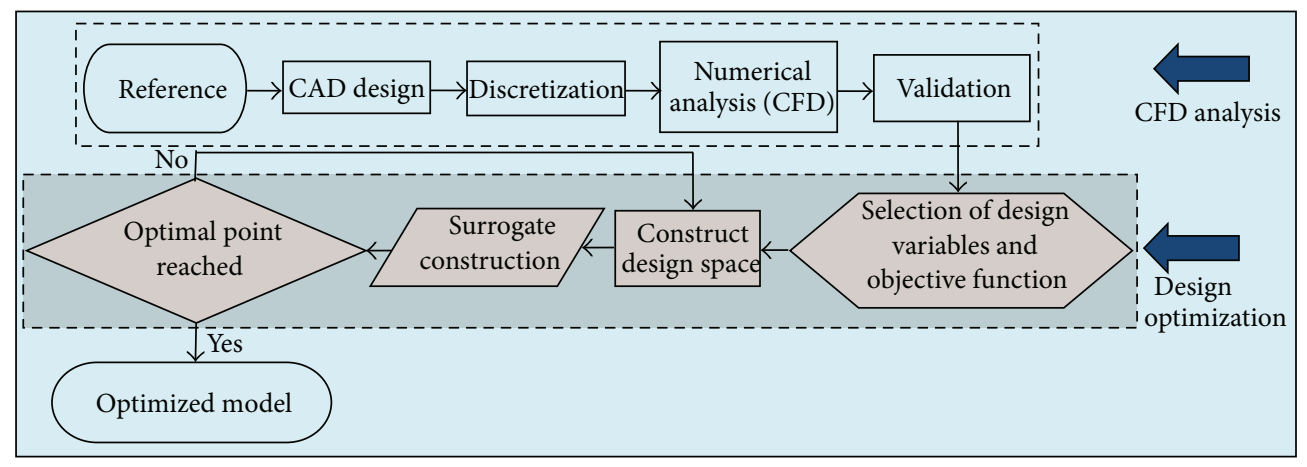

FIgURE 3: Optimization procedure.

TABle 3: Design space.

\begin{tabular}{lcc}
\hline Variables & LB & UB \\
\hline$N_{\mathrm{rb}}$ & 30 & 42 \\
$N_{\mathrm{gv}}$ & 20 & 32 \\
\hline
\end{tabular}

2.4. Surrogate Model Construction and Search Algorithm. The surrogates such as KRG variants and RSA functions mimic a high-fidelity response. Kriging variants are approximation functions with multiple inputs and a single output. The UKR is basically a data fitting or an interpolating technique that uses a trend function or a regression function to capture large-scale variations and a systematic departure or stochastic process $(x)$ through the residuals to capture small-scale variations. Depending on the nature of the regression function Kriging variants have been organized with various names [13]. Simple Kriging presumes the regression function to be a known constant; that is, $f(x)=0$. A popular category is OKR which imagines a constant but unknown regression function $f(x)=\mu$. The BKR deploys the linear regression function as a trend function. In another class, UKR regards the trend function as multivariate polynomial.

Kriging is also considered as Gaussian process, and a summary of the same is presented below $[4,13,14]$. The " $n$ " training points can be represented as $x_{1}, x_{2}, \ldots, x_{n}$, and let $f(X)$ represent the response. The Kriging interpolation is derived as

$$
\tilde{f}(x)=M \alpha+r(x) \cdot \Psi^{-1} \cdot(f(X)-F \alpha) .
$$

Here, $M$ and $F$ represent the model matrices of the test point $x$ and the training set $X$, respectively. The regression coefficient function $\alpha$ is determined by method of generalized least squares:

$$
\alpha=\left(X^{\prime} \Psi^{-1} X\right)^{-1} X \Psi^{-1} f(X)
$$

$r(\mathbf{x})=\left(\psi\left(\mathbf{x}, \mathbf{x}^{1}\right), \ldots, \psi\left(\mathbf{x}, \mathbf{x}^{n}\right)\right)$ is an $1 \times n$ vector of correlations between the data point $x$ and training set $X$. $\Psi$ is $n \times n$ correlation matrix given as

$$
\Psi=\left(\begin{array}{ccc}
\psi\left(\mathbf{x}^{1}, \mathbf{x}^{1}\right) & \cdots & \psi\left(\mathbf{x}^{1}, \mathbf{x}^{n}\right) \\
\vdots & \ddots & \vdots \\
\psi\left(\mathbf{x}^{n}, \mathbf{x}^{1}\right) & \cdots & \psi\left(\mathbf{x}^{n}, \mathbf{x}^{n}\right)
\end{array}\right) .
$$

The regression function may be considered as mean of the geometric progression. The prediction which is far from data point will be reverted to the mean. Initially, the behavior of the response is unknown; hence a constant regression function $\alpha=\mu$ is assigned; this interpolation methodology describes ordinary Kriging (OKR). Also, it is possible by applying prior knowledge or any other technique, to identify the basis functions which could be used in the regression function. This enables extrapolating the points outside the sampled region. If a quadratic polynomial is used, the Kriging technique is termed as universal Kriging (UKR), whereas when the Kriging is able to identify the best basis function on its own, it is termed as blind Kriging (BKR). The importance of each basis function is determined using the Bayesian variable ranking. Further, forward selection strategy is applied to enable inclusion of more basis functions in the BKR model. The merit of using this method is that it satisfies principles of effect hierarchy and effect heredity; for example, linear interactions are included before the quadratic effects.

RSA is a methodology [14] of fitting a function for discrete responses obtained from numerical calculations. For a second-order polynomial RSA model, the response can be represented by

$$
\begin{aligned}
F(x)= & \theta_{o}+\sum_{j=1}^{N_{\mathrm{DV}}} \theta_{j} x_{j} \\
& +\sum_{j=1}^{N_{\mathrm{DV}}} \theta_{j j} x_{j}^{2}+\sum_{i=1}^{N_{\mathrm{DV}}} \sum_{\substack{j=1 \\
i \neq j}}^{N_{\mathrm{DV}}} \theta_{i j} x_{i} x_{j},
\end{aligned}
$$

where $F(x)$ represents the response of the function, and terms $\theta_{o}, \theta_{1}$, and so forth are the regression coefficients. The number of regression coefficients are found by relation 
$\left(N_{\mathrm{DV}}+1\right) x\left(N_{\mathrm{DV}}+2\right) / 2$, where $N_{\mathrm{DV}}$ is the number of design variables and $x$ represents the selected design variable. It is the simplest model and most commonly used in the surrogate based optimization application.

GA [15], a population-based algorithm, is used for the global optimal search. As GA is based on random number generation, each run produces a different result, and actual local optima can be ignored. Hence, in order to reduce the problem of GA, sequential quadratic programming (SQP), which is a local search algorithm, was used for fine-tuning. SQP can be used directly to search optimally, but it is dependent on initial guesses for the design variables as to where the global optimum can be located. A highly nonlinear function can have several local optima, and different guesses produce different designs. One option for alleviating that issue is to use several initial guesses, run SQP several times, and choose the best objective function value among the predicted responses. Another solution is using hybrid GA, searching for a global optimum with GA, and then fine-tuning with SQP.

GA function in Matlab takes the following parameters: surrogate function to generate population, number of initial population, boundary of the variables, and stopping criteria. Initially 20 populations were generated and number of generations was set to 50 . Once the iteration is complete, the optimal design variable values were obtained. On the other hand in SQP, initial guess along with the boundary of the variables was fed. In this case, the optimal design variable values were used as initial guess of SQP. Each run of GA produces different result because it tries to find global optimal while with proper initial guess SQP finds a local optima or best design.

\section{Results and Discussions}

Figure 4 validates the current CFD result with existing experimental and CFD results for the objective function [10, 16]. In the present simulations, 1.4 million cell elements and 0.275 million nodes were generated. Design points in the design space were selected through three-level full factorial design. The objective function which is turbine efficiency was evaluated at these points using the RANS solver. The evaluated objective function values are shown in Figure 5. The computed efficiency values along with the design points were used for the surrogate construction. Finally, the HGA was used to find optimal points from the surrogates. The optimal design is solved again using the RANS solver to check the accuracy of surrogates.

Table 4 shows the comparison of RANS and surrogate predicted results of the objective function. The optimized results show that the efficiency has increased to $42.52 \%$ which was around $12.67 \%$ increment as compared to the reference design and it was obtained by UKR. The error in prediction of UKR was $-0.31 \%$ which was lowest among all the surrogates considered for the present evaluation. The error was because of the noninteger prediction of the number of blades, approximation in CFD computations, and surrogate construction. The table shows the optimal point in the design space and the optimal numbers of RB and GV were 38 and 24, respectively.

As the efficiency was highest by the UKR predicted result, further study to analyze the flow was done using the UKR

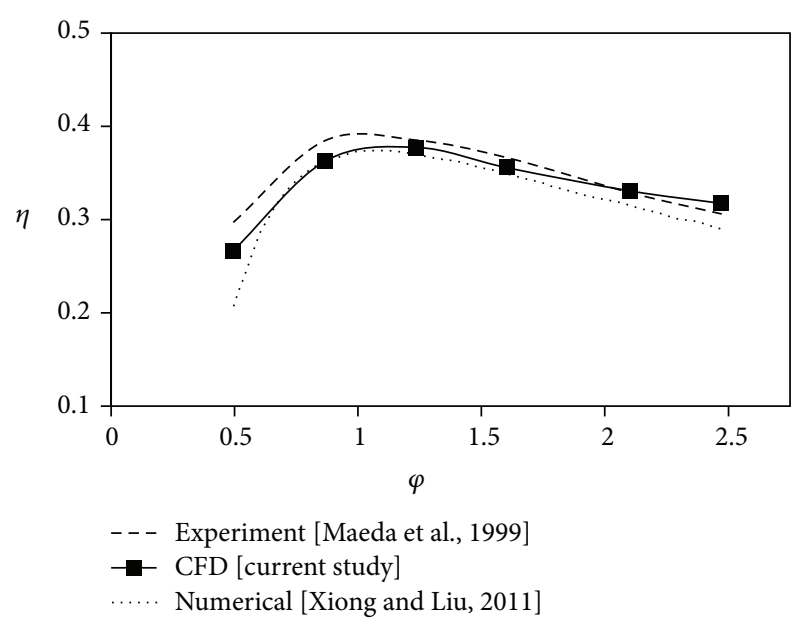

FIGURE 4: Validation for objective function.

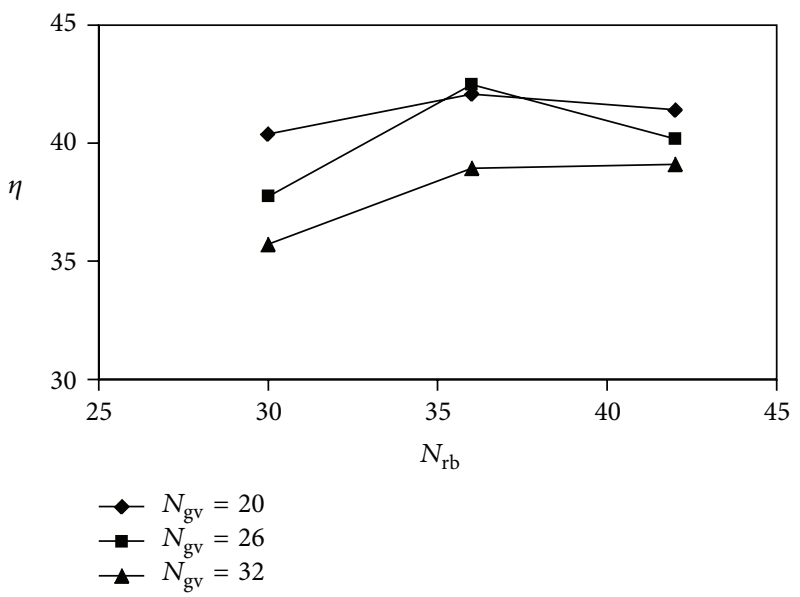

FIGURE 5: Objective function values.

predicted results only. Figures 6-9 show the comparison of Ref and Opt designs. For a wider range of flow coefficient, the Opt design shows the overall improvement in the efficiency (Figure 6). The power produced is higher for the Opt design which contributes to higher torque (Figure 7). A turbine should produce lesser pressure drop, which gives higher efficiency, and this can be clearly observed for the Opt design in Figure 8.

Figure 9 shows the pressure distribution at a plane located at $50 \%$ span for the base and the optimal design. It was observed that the pressure contour over the blade is lower for the optimized blade. The pressure over the rotor is higher for the reference design and is lower for the optimum design, which results in lesser pressure drop across the optimum blade. The lesser the pressure drop is, the more it contributes to efficiency.

Among the surrogates, it was found that the UKR performed well while the BKR performed badly. The most general surrogate RSA somehow improved efficiency but error was higher compared to UKR but lower than BKR. Similar confusing results were found by the authors $[2,5,6]$ 
TABle 4: Optimal designs for efficiency.

\begin{tabular}{lccccccc}
\hline Model & $N_{\mathrm{gv}, \mathrm{opt}}$ & $N_{\text {rb,opt }}$ & $F_{\text {Sur }}, \%$ & $F_{\text {CFD }}, \%$ & $F_{\text {Ref }}, \%$ & $F_{\text {Imp }}, \%$ & -0.53 \\
\hline BKR & 25 & 37 & 42.67 & 37.54 & 37.74 & -13.67 \\
OKR & 24 & 36 & 42.89 & 42.07 & 37.74 & 11.47 & -1.95 \\
UKR & 24 & 38 & 42.65 & 42.52 & 37.74 & -0.31 \\
RSA & 20 & 37 & 42.71 & 41.09 & 37.74 & -3.67 & 8.88 \\
\hline
\end{tabular}

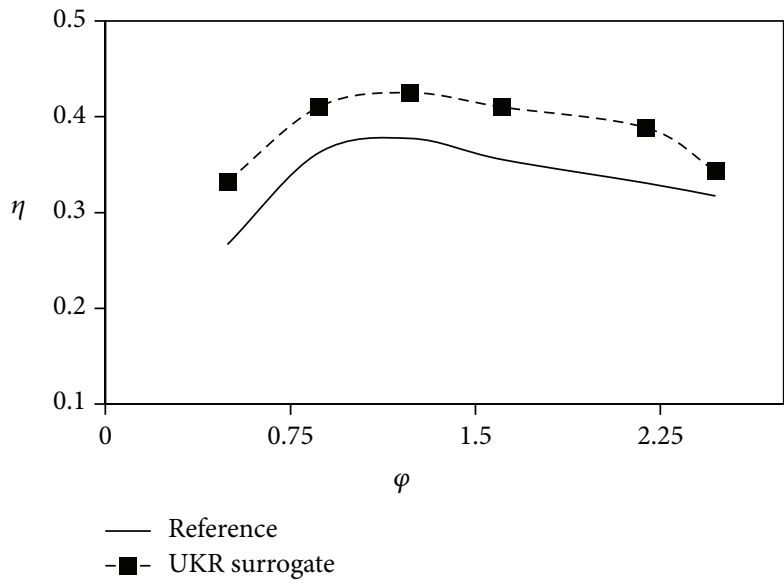

FIgURE 6: Efficiency enhancement.

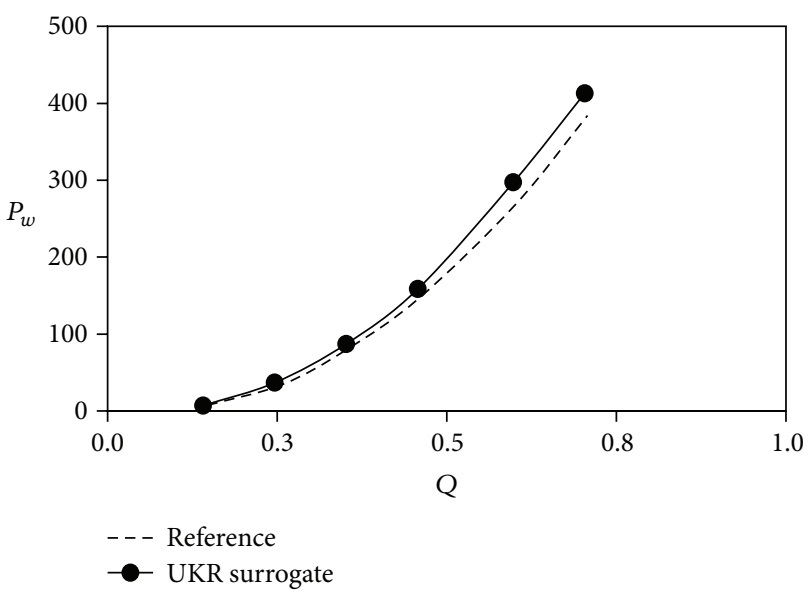

FiguRE 7: Effect of flow rate on power.

for the surrogates RSA, OKR, and neural network. Hence a multiple surrogate approach is better for turbomachinery applications as the same set of design points can produce multiple optimal and there is a greater chance to have better optimal design and less uncertainty in optimal design.

\section{Conclusion}

An impulse turbine was numerically modeled and analyzed using a RANS solver. Different surrogate models including Kriging variants and RSA were used to find optimal design. The optimizer was hybrid genetic algorithm. It was found

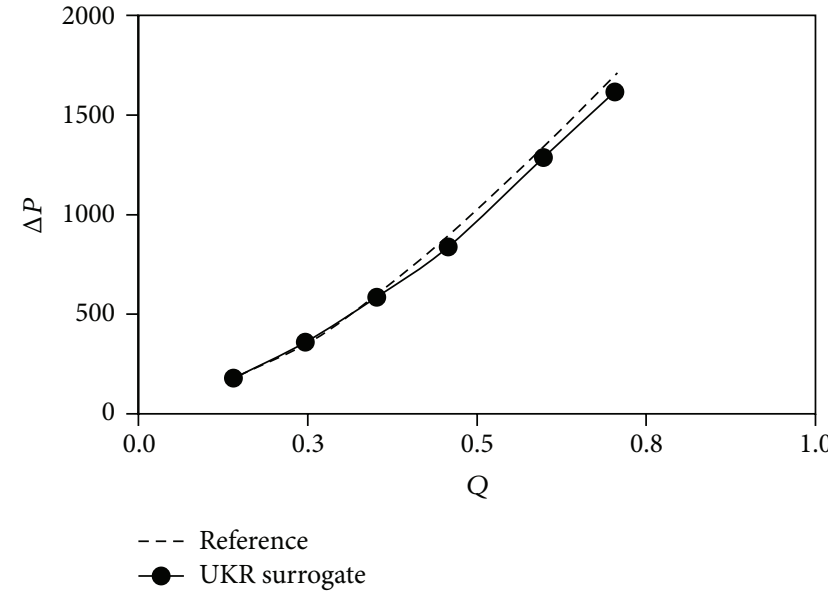

FIGURE 8: Effect of flow rate on pressure drop.

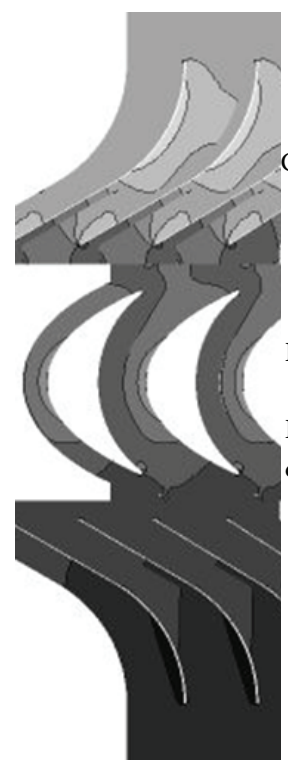

Reference
GV upstream

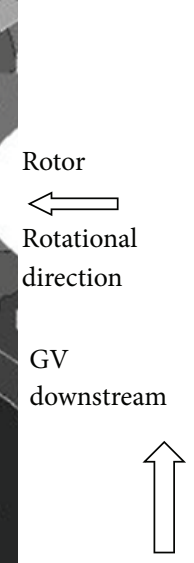

Flow direction

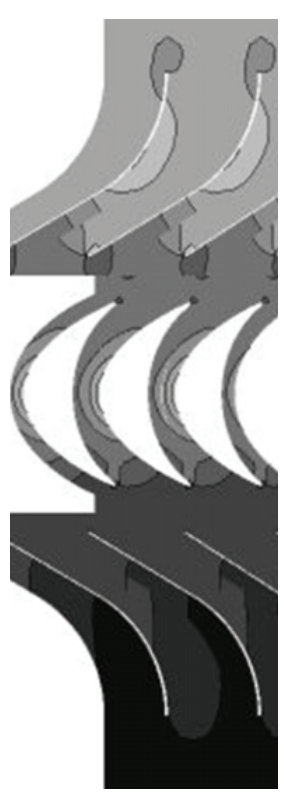

Optimized

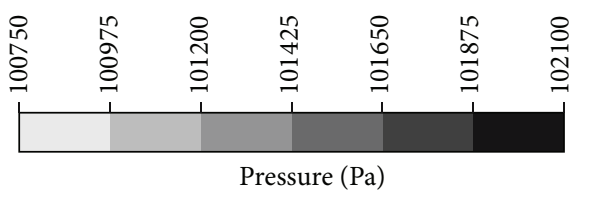

Figure 9: Pressure contour. 
that approximately $13 \%$ relative efficiency can be improved through the optimization procedure. The optimal number of rotor and stator blades was 38 and 24. The increment in efficiency was significant over the entire flow coefficients. The enhancement of efficiency was achieved because of change in pressure profile over the blade.

Among the different surrogates universal Kriging performed better while blind Kriging failed to enhance the turbine performance. Hence, instead of single surrogate, multiple surrogate application is suggested to the readers. The CFD with the surrogate coupled hybrid genetic algorithm can be used for the ocean energy applications as this approach can reduce total design and simulation cost.

\section{Nomenclature}

\section{Abbreviations}

BKR: Blind Kriging

CFD: Computational fluid dynamics

DV: Design variable

GA: Genetic algorithm

GV: Guide vane

KRG: Kriging

OKR: Ordinary Kriging

UKR: Universal Kriging

Opt: Optimized

OWC: Oscillating water column

RANS: Reynolds averaged Navier-Stoke

RB: $\quad$ Rotor blade

Ref: Reference

RSA: Response surface approximation

SQP: Sequential quadratic programming.

\section{Symbols}



Subscripts

cfd: Computational fluid dynamics

DV: Design variables
Err: Error

gv: Guide vane

imp: Improvement

in: Input

Opt: Optimal

Ref: Reference

rb: Rotor blade

$s$ : Rotational speed

Sur: Surrogate

$t$ : Torque.

\section{Conflict of Interests}

The authors do not have any conflict of interests.

\section{Acknowledgments}

The authors gratefully acknowledge the financial support by the Earth System Science Organization, Ministry of Earth Sciences, Government of India, to conduct the research.

\section{References}

[1] T. D. Robinson, Surrogate based optimization using multifidelity models with variable parameterization [Ph.D. thesis], Massachusetts Institute of Technology, Cambridge, Mass, USA, 2007.

[2] A. Samad, K.-Y. Kim, T. Goel, R. T. Haftka, and W. Shyy, "Multiple surrogate modeling for axial compressor blade shape optimization," Journal of Propulsion and Power, vol. 24, no. 2, pp. 302-310, 2008.

[3] F. A. C. Viana, T. W. Simpson, V. Balabanov, and V. Toropov, "Metamodeling in multidisciplinary design optimization: how far have we really come?" AIAA Journal, vol. 52, no. 4, pp. 670 690, 2014.

[4] J. Peter and M. M. Onera, "Comparison of surrogate models for turbomachinery design," WSEAS Transactions on Fluid Dynamics, vol. 3, no. 1, pp. 10-17, 2008.

[5] A. Samad, Numerical optimization of turbomachinery blade using surrogate models [Ph.D. thesis], School of Mechanical Engineering, Inha University, Incheon, South Korea, 2008.

[6] A. Samad and K.-Y. Kim, "Shape optimization of an axial compressor blade by multi-objective genetic algorithm," Proceedings of the Institution of Mechanical Engineers, Part A: Journal of Power and Energy, vol. 222, no. 6, pp. 599-611, 2008.

[7] A. F. D. O. Falcão, "Wave energy utilization: a review of the technologies," Renewable and Sustainable Energy Reviews, vol. 14, no. 3, pp. 899-918, 2010.

[8] M. H. Mohamed, G. Janiga, E. Pap, and D. Thévenin, "Multiobjective optimization of the airfoil shape of Wells turbine used for wave energy conversion," Energy, vol. 36, no. 1, pp. 438-446, 2011.

[9] R. P. F. Gomes, J. C. C. Henriques, L. M. C. Gato, and A. F. O. Falcão, "Multi-point aerodynamic optimization of the rotor blade sections of an axial-flow impulse air turbine for wave energy conversion," Energy, vol. 45, no. 1, pp. 570-580, 2012.

[10] H. Maeda, S. Santhakumar, T. Setoguchi, M. Takao, Y. Kinoue, and K. Kaneko, "Performance of an impulse turbine with fixed guide vanes for wave power conversion," Renewable Energy, vol. 17 , no. 4, pp. 533-547, 1999. 
[11] A. Thakker and T. S. Dhanasekaran, "Experimental and computational analysis on guide vane losses of impulse turbine for wave energy conversion," Renewable Energy, vol. 30, no. 9, pp. 1359-1372, 2005.

[12] T. Setoguchi, S. Santhakumar, H. Maeda, M. Takao, and K. Kaneko, "A review of impulse turbines for wave energy conversion," Renewable Energy, vol. 23, no. 2, pp. 261-292, 2001.

[13] I. Couckuyt, A. Forrester, D. Gorissen, F. De Turck, and T. Dhaene, "Blind Kriging: implementation and performance analysis," Advances in Engineering Software, vol. 49, no. 1, pp. 1-13, 2012.

[14] R. H. Myers and D. C. Montgomery, Response Surface Methodology-Process and Product Optimization Using Designed Experiments, John Wiley \& Sons, New York, NY, USA, 1995.

[15] D.-K. He, F.-L. Wang, and Z.-Z. Mao, "Hybrid genetic algorithm for economic dispatch with valve-point effect," Electric Power Systems Research, vol. 78, no. 4, pp. 626-633, 2008.

[16] C. Xiong and Z. Liu, "Numerical analysis on impulse turbine for OWC wave energy conversion," in Proceedings of the AsiaPacific Power and Energy Engineering Conference (APPEEC '11), Wuhan, China, March 2011. 





The Scientific World Journal


Submit your manuscripts at http://www.hindawi.com
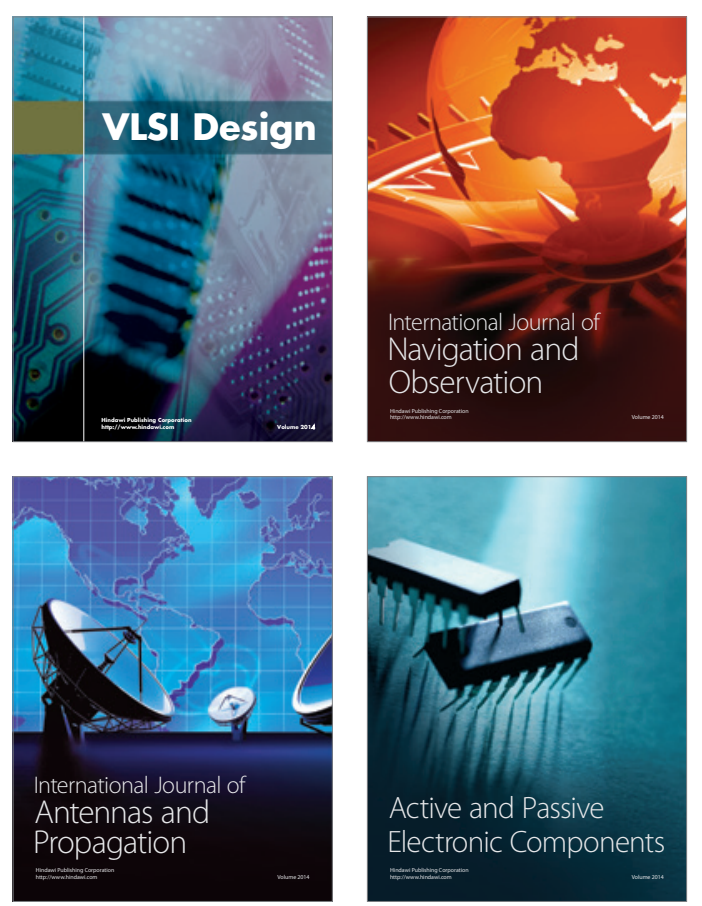
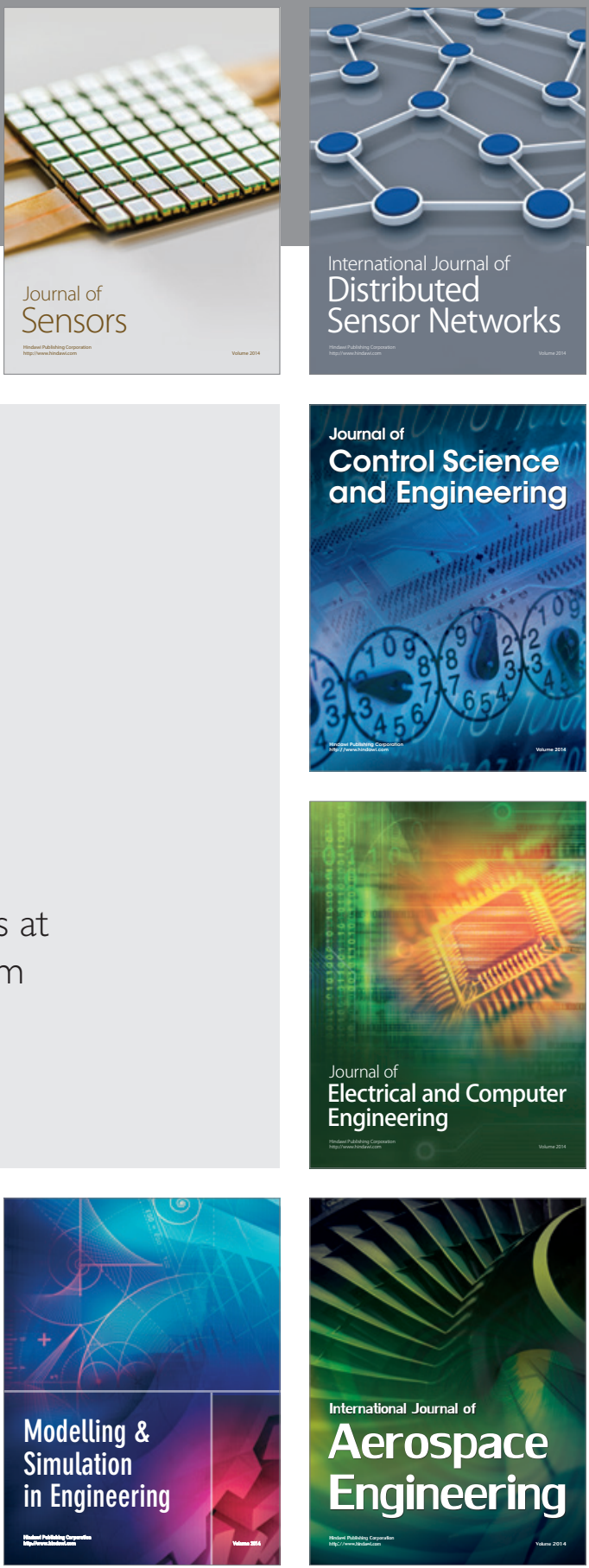

Journal of

Control Science

and Engineering
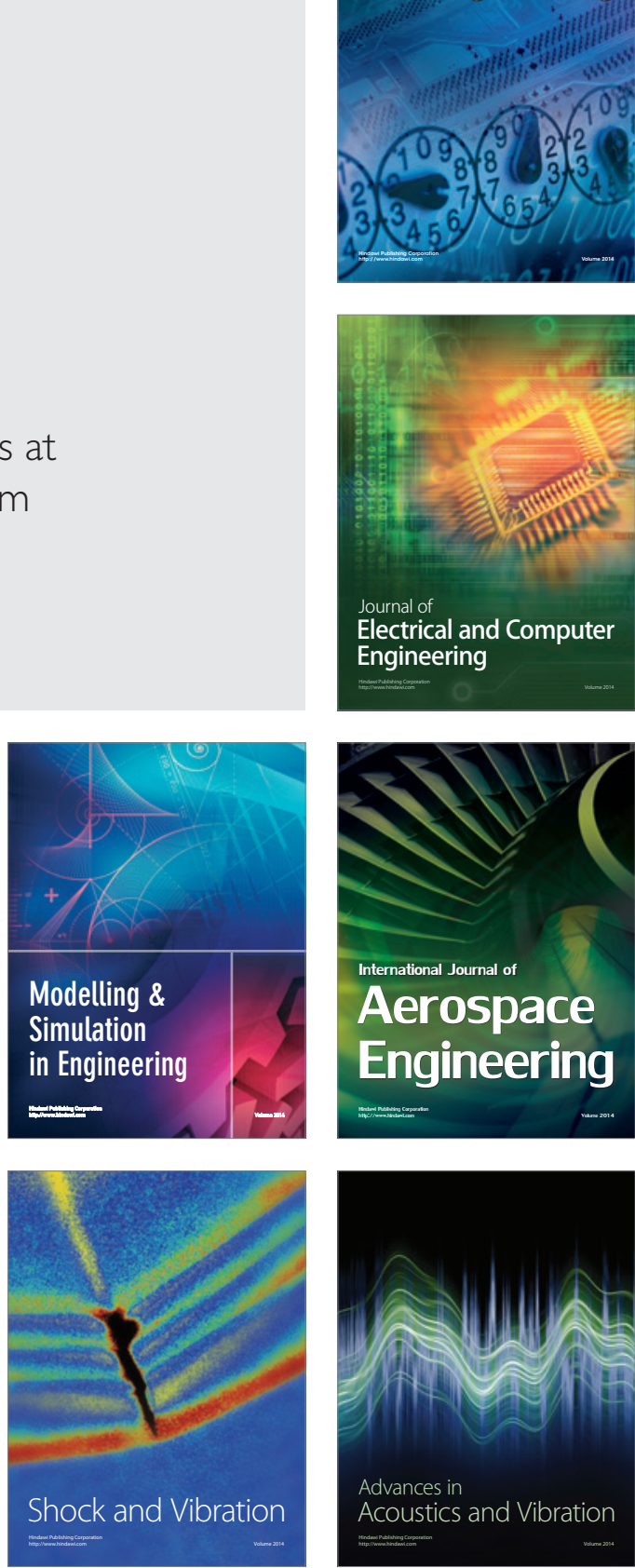